\title{
Broken Bar Fault Detection based on Set Membership Identification for Three Phase Induction Motors
}

\author{
Mohammed Obaid Mustafa ${ }^{1}$, George Nikolakopoulos ${ }^{1}$, Thomas Gustafsson ${ }^{1}$ and Basil M. Saied ${ }^{2}$ \\ ${ }^{1}$ Department of Computer Science, Electrical and Space Engineering, Division of Systems and Interaction, \\ Luleå University of Technology, Luleå, Sweden \\ ${ }^{2}$ Department of Electrical Engineering, University of Mosul, Mosul, Iraq
}

Keywords: Three Phase Induction Motor, Fault Detection, Set Membership Identification.

\begin{abstract}
This article presents a fault detection scheme for the case of a broken bar occurrence in a three phase induction motor. The proposed scheme relies on Set Membership Identification (SMI) and novel proposed boundary violation rules for the identified motor's parameters. The model of the three phase induction motor is being transformed into an equivalent two phase model, described in the $q-d$ space, for both the normal and the faulty case. By the utilization of the SMI technique, the simplified equivalent model of the induction motor is being identified during the steady state operation (non-fault case), while at the same time safety bounds for the identified variables are being provided, based on an a priori defined corrupting additive noise. On the event of a fault, specific fault detection conditions are being proposed that can capture the specific type of a broken bar fault. The proposed conditions depend on: a) abnormal parameter jumps, and b) rapid changes in the volume of the bounding uncertainty, which is being formulated either by ellipsoids or orthotopes. Detailed analysis of the proposed approach as also extended simulation results are being presented that prove the efficiency of the proposed scheme.
\end{abstract}

\section{NOMENCLATURE}

$V_{s a}, V_{s b}, V_{s c}$ : Stator's three phase voltages $(V)$

$V_{r a}, V_{r b}, V_{r c}$ : Rotor's three phase voltages $(V)$

$i_{r a}, i_{r b}, i_{r c}$ : Rotor's three phase currents $(A)$

$i_{s a}, i_{s b}, i_{s c}:$ Stator's three phase currents $(A)$

$r_{s}, r_{r}$ : Resistance of stator's and rotor's winding $(\mathrm{Ohm})$

$L_{s s}, \quad L_{r r}:$ Stator's and rotor's self-inductances (Henry)

$L_{S}, L_{r}$ : Stator's and rotor's self inductance (Henry)

$L_{m}$ : Mutual inductance (Henry)

$\omega_{r}$ : Rotor's angular speed $(\mathrm{rad} / \mathrm{sec})$

$\omega_{m}$ : Rotor's speed (mechanical) $(\mathrm{rad} / \mathrm{sec})$

$\omega_{s}$ : Supply angular frequency $(\mathrm{rad} / \mathrm{sec})$

$P$ : No. of poles pairs

$J$ : Moment of inertia $\left(\mathrm{Kg} \cdot \mathrm{m}^{2}\right)$

$T_{L}:$ Load torque $(\mathrm{Nm})$

$T_{e}:$ Electromagnetic torque $(\mathrm{Nm})$

$q$ : Quadrature axis frame

$d$ : Direct axis frame

$s$ : Stator quantities

$r:$ Stator quantities

$\Psi_{s}, \Psi_{r}$ : Stator's and Rotor's fluxes (Weber) $\theta$ : Angular position in the frame of motor (Deg)

$\theta_{r}$ : Angle between rotor's phase axis and stator's phase axis

$\beta$ : Angle between rotor's phase axis and stator's phase axis

$N_{b}$ : Numbers of machine rotor bars

\section{INTRODUCTION}

The three phase induction motor is one of the most important types of motors that are commonly being found in many industrial applications, mainly due to their efficiency and reliability (Chen and Zivanovic, 2009), due to the fact that these motors are operating in difficult working environments with many factors that degrade their performance such as: dust, temperature level, humidity, continuous operation, and heavy loads, internal faults frequently occur.

The most common faults that could be appeared in the rotor and the stator of an induction motor are: a) short circuit stator winding (Nandi and Toliyat, 2005), b) broken rotor bars (Santos and Lubiny, 2010), c) bearing failures, and d) dynamic or static air gab ir- 
regularities (Nandi and Toliyat, 2005). From all these types of faults, the broken bar faults comprise around $(5-10) \%$ of all the reported motor faults, while it is necessary to detect this type of fault, as soon as possible as these type of faults can add serious motor damage if not detected on time.

In general for detecting the mechanical or electrical faults in a three phase induction motor multiple methods have been proposed, which can be categorized in direct and indirect methods. Direct methods base their operation on spectral analysis of stator currents, stator voltages, and electromagnetic torque (Bachi et al., 2006), with the focus to be on detecting spectrum lines at certain frequencies using classical methods like Fouriers analysis and are quite simple to be implemented. A significant drawback of these methods is the fact that these are best suited for fixed speed applications, while in industrial applications under varying speed and with a direct power supply, these methods are not well adapted due to the fact that the involved electrical signals are not stationary. Indirect methods base their operation on identification or prediction techniques and multiple identification schemes for fault detection and fault diagnosis have been appeared in the literature.

In parallel to these indirect fault detection schemes, Set Membership Identification (SMI) (Deller, 1989; Deller et al., 1993) has received a growing attention in the past years as a quite important technique for system identification with uncertainty bounds. The main novelty of this article stems from the adaptation of the SMI approach to the problem of fault detection and more specifically to the problem of detecting broken rotor bar fault for an induction motor. To the author's best knowledge this is the first time that such an approach is being reported in the scientific literature. The extension of this scheme to other types of faults can support a general fault detection framework, where fault diagnosis could be also performed in parallel with the fault detection scheme. Based on the proposed approach, the three phase model of the induction motor is being transformed to an equivalent two phase model for the healthy and the faulty case, and the safety intervals for the online SMI for the identified parameters, are establishing a robust fault detection scheme that could be directly transferred to real-life implementations.

The rest of the article is being structured as it follows. In Section 2 the model derivation and simplification, for the healthy and the faulty cases are being derived. In Section 3 the SMI scheme is being presented, followed by the proposed fault detection conditioning framework in Section 4. Section 5 contains multiple simulation results that prove the efficacy of the proposed methodology, while the conclusions are drawn in the last Section 6.

\section{INDUCTION MOTOR MODELING}

\subsection{Healthy Case}

In general an induction motor can be modeled as a three phase model or as an equivalent quadrature phase model, while the voltage balance equations for the case of three phases can be formulated as (Vas, 1992):

$$
V=p \psi+\mathbf{R} \mathbf{i}
$$

where

$$
\begin{aligned}
& \mathbf{V}=\left[\begin{array}{llll}
V_{s a} V_{s b} V_{s c} V_{r a} V_{r b} V_{r c}
\end{array}\right]^{T} \\
& \psi=\left[\psi_{s a} \psi_{s b} \psi_{s c} \psi_{r a} \psi_{r b} \psi_{r c}\right]^{T} \\
& \mathbf{i}=\left[\begin{array}{llllll}
i_{s a} & i_{s b} & i_{s c} & i_{r a} & i_{r b} & i_{r c}
\end{array}\right]^{T} \\
& \mathbf{R}=\operatorname{diag}\left[r_{s} r_{s} r_{s} r_{r} r_{r} r_{r}\right]
\end{aligned}
$$

and $r_{s a}=r_{s b}=r_{s c}$ and $r_{r a}=r_{r b}=r_{r c}$ in the balance case of motor, the operator $p$ is equal to $d / d t$ and the equations of three phase input voltages are:

$$
\begin{aligned}
& V_{s a}=V_{m} \sin \left(\omega_{s} t\right) \\
& V_{s b}=V_{m} \sin \left(\omega_{s} t-2 \pi / 3\right) \\
& V_{s c}=V_{m} \sin \left(\omega_{s} t-4 \pi / 3\right)
\end{aligned}
$$

and the relation between the phase linkages and the phase currents is provided by:

$$
\psi=\mathbf{L} \mathbf{i}
$$

or

$$
\mathbf{L} p \mathbf{i}=\mathbf{V}-\mathbf{R} \mathbf{i}
$$

In this formulation the values of the inductance matrix $\mathbf{L}$ depend on the rotor's electrical angle and the type of the utilized model (Chen and Zivanovic, 2009). For simplifying the three phase model, the equivalent two phase model will be extracted that has been converted to the $q-d$ coordination frame (Sandhu and Pahwa, 2009). For predicting the mechanical and electrical behavior of the original machine correctly, the original $a b c$ variables $F_{a b c}$ should be transformed into the corresponding $d-q$ variables $F_{q d o}$ and this is being carried out through Park's transform as it follows (Lee et al., 1985):

$$
\begin{aligned}
\mathbf{F}_{\text {dqo }} & =\mathbf{T}_{\text {qdo }} \cdot \mathbf{F}_{\text {abc }} \\
\mathbf{V}_{\text {dqo }} & =\mathbf{T}_{\text {qdo }} \cdot \mathbf{V}_{\text {abc }} \\
\mathbf{i}_{\text {dqo }} & =\mathbf{T}_{\text {qdo }} \cdot \mathbf{i}_{\text {abc }}
\end{aligned}
$$


where

$$
\mathbf{T}_{\mathbf{d q o}}=\frac{\mathbf{2}}{\mathbf{3}}\left[\begin{array}{ccc}
\cos \theta & \cos \theta_{1} & \cos \theta_{2} \\
\sin \theta & \sin \theta_{1} & \sin \theta_{2} \\
1 / 2 & 1 / 2 & 1 / 2
\end{array}\right]
$$

and

$$
\begin{aligned}
\mathbf{V}_{\text {dqo }} & =\left[\begin{array}{lll}
v_{q} & v_{d} & v_{0}
\end{array}\right]^{T} \\
\mathbf{i}_{\mathbf{d q o}} & =\left[\begin{array}{lll}
i_{q} & i_{d} & i_{0}
\end{array}\right]^{T}
\end{aligned}
$$

with $\theta_{1}=\theta-2 \pi / 3, \theta_{2}=\theta-4 \pi / 3$. In the case that the saturation and the fraction effects are being neglected, the balance voltage equation of the three phase induction motor in $q-d$ model are provided by:

$$
\mathbf{L}_{\mathbf{q d}} \mathbf{p} \mathbf{i}_{\mathbf{q d}}^{\mathrm{sr}}=\mathbf{V}_{\mathbf{q d}}^{\mathrm{sr}}-\mathbf{R}_{\mathbf{q d}} \mathbf{i}_{\mathbf{q d}}^{\mathrm{sr}}
$$

and the $\mathbf{p i}_{\mathbf{q d}}^{\mathbf{s r}}$ can be calculated as:

$$
\mathbf{p i} \mathbf{q d}_{\mathbf{q}}^{\mathbf{s}}=-\mathbf{L}_{\mathbf{q d}}^{-1} \mathbf{R}_{\mathbf{q d}} \mathbf{i}_{\mathbf{q d}}^{\mathrm{sr}}+\mathbf{L}_{\mathbf{q d}}^{-1} \mathbf{V}_{\mathbf{q d}}^{\mathrm{sr}}
$$

where

$$
\begin{aligned}
\mathbf{V}_{\mathbf{q d}}^{\mathbf{s r}}= & {\left[\begin{array}{llll}
v_{q s} & v_{d s} & v_{q r} & v_{d r}
\end{array}\right]^{T} } \\
\mathbf{i}_{\mathbf{q d}}^{\mathbf{s r}}= & {\left[\begin{array}{llll}
i_{q s} & i_{d s} & i_{q r} & i_{d r}
\end{array}\right]^{T} } \\
\mathbf{L}_{\mathbf{q d}}= & {\left[\begin{array}{cccc}
L_{s} & 0 & L_{m} & 0 \\
0 & L_{s} & 0 & L_{m} \\
L_{m} & 0 & L_{r} & 0 \\
0 & L_{m} & 0 & L_{r}
\end{array}\right] }
\end{aligned}
$$

and

$$
\mathbf{R}_{\mathbf{q d}}=\left[\begin{array}{cccc}
r_{s} & 0 & 0 & 0 \\
0 & r_{s} & 0 & 0 \\
0 & -\omega_{r} / \omega_{s} L_{m} & r r & -\omega_{s} / \omega_{s} L_{r} \\
\omega_{r} / \omega_{s} L_{m} & 0 & \omega_{r} / \omega_{s} L_{r} & r r
\end{array}\right]
$$

After these formulations, equation (10) can be rewritten in a state space form as:

$$
\left[\begin{array}{c}
\frac{d i_{q s}}{d t} \\
\frac{d i_{d s}}{d t} \\
\frac{d i_{q r}}{d t} \\
\frac{d i_{d r}}{d t}
\end{array}\right]=\mathbf{A}\left[\begin{array}{c}
i_{q s} \\
i_{d s} \\
i_{q r} \\
i_{d r}
\end{array}\right]+\mathbf{B}\left[\begin{array}{c}
V_{q s} \\
V_{d s} \\
0 \\
0
\end{array}\right]
$$

where:

$$
\begin{aligned}
& \mathbf{A}=-\mathbf{R}_{\mathbf{q d}} \mathbf{L}_{\mathbf{q d}}{ }^{-1} \\
& \mathbf{B}=\mathbf{L}_{\mathbf{q d}}{ }^{-1}
\end{aligned}
$$

and with the $\mathbf{A}$ and $\mathbf{B}$ matrices defined as:

$$
\begin{aligned}
\mathbf{A} & =\frac{1}{\delta}\left[\begin{array}{cccc}
-L_{r} r_{s} & 0 & L_{m} r_{s} & 0 \\
0 & -L_{r} r_{s} & 0 & L_{m} r_{s} \\
L_{m} r_{r} & 0 & -L_{s} r_{r} & w_{r} \delta \\
0 & L_{m} r_{r} & -w_{r} \delta & -L_{s} r_{r}
\end{array}\right] \text { (18) } \\
\mathbf{B} & =\frac{1}{\delta}\left[\begin{array}{cccc}
L_{r} & 0 & -L_{m} & 0 \\
0 & L_{r} & 0 & -L_{m} \\
-L_{m} & 0 & \mathrm{E}_{s} & 0 \\
0 & -L_{m} & 0 & L_{s}
\end{array}\right]
\end{aligned}
$$

while $\delta$ is given as:

$$
\delta=L_{s} L_{r}-L m^{2}
$$

Finally, the resulting equations for the torque and the mechanical angular speed for the three phase induction motor are:

$$
\begin{aligned}
T_{e} & =\frac{3}{2} P L_{m}\left[i_{q s} i_{d r}-i_{q r} i_{d s}\right] \\
d \omega_{m} & =\frac{1}{J}\left[T_{e}-T_{L}\right] d t
\end{aligned}
$$

\subsection{Broken Bar Model of Three Phase Induction Motor}

The focus of this research effort is on the identification of broken bar faults in the rotor of a three phase induction motor. The reasons of broken bar faults are thermal, magnetic, residual, dynamic, and mechanical stresses (Nandi and Toliyat, 2005). The event of a broken rotor bar causes asymmetry of the resistance and inductance in rotor phases, which results in asymmetry of the rotating electromagnetic field in the air gap between stator and rotor. Consequently, this will induce frequency harmonics in the stator current. The impact of broken rotor bars can be modeled by unbalancing the rotor resistance, while the inductance changes are being neglected due to their insignificance influence compared to the resistance changes (Bellini et al., 2001; Chen and Zivanovic, 2009), and the stator resistances and inductances stay unchanged (Chen and Zivanovic, 2009). For simplicity, for a squirrel-cage rotor, the end-ring contribution is being also neglected.

In the case of such a fault, the modified (faulty) versions of matrices $\mathbf{A}^{*}$ should be utilized. The modified rotor resistance matrix in the $a b c$ reference frame can be derived as (Chen and Zivanovic, 2009):

$$
\mathbf{r}_{\mathbf{r f}}=\left[\begin{array}{ccc}
r_{r}+\Delta r_{r a} & 0 & 0 \\
0 & r_{r}+\Delta r_{r b} & 0 \\
0 & 0 & r_{r}+\Delta r_{r c}
\end{array}\right]
$$

where $\Delta r_{r a}, \Delta r_{r b}$ and $\Delta r_{r c}$ represent rotor resistance changes in phase $\mathrm{a}, \mathrm{b}$ and $\mathrm{c}$, respectively due to broken bar faults. The resistance changes are being derived based on the assumption that the broken bars are contiguous, neither the end ring resistance nor the magnetizing current is taken into account. For the case of $n_{b b}$ broken rotor bars, the increment $\Delta r_{r a, b, c}$ in each phase is being obtained as (Chen and Zivanovic, 2009; Kumar et al., 2011).

$$
\Delta r_{r a, b, c}=r_{r} \frac{3 n_{b b}}{N_{b}-3 n_{b b}}
$$

To simulate broken rotor bar fault, the rotor resistance matrix needs to be replaced by the modified rotor resistance matrix $r_{r f}$ and transformed to $q-d$ reference 
frame, by utilizing the stationary reference frame. For the cased examined, the rotor resistance changes in the $q-d$ reference frame as (Chen and Zivanovic, 2009).

$$
\Delta r_{r f}^{q d 0}=\left[\begin{array}{lll}
r_{r 11} & r_{r 12} & r_{r 13} \\
r_{r 21} & r_{r 22} & r_{r 23} \\
r_{r 31} & r_{r 32} & r_{r 33}
\end{array}\right]
$$

where

$$
\begin{aligned}
r_{r 11}= & \frac{1}{3}\left(\Delta r_{r a}+\Delta r_{r b}+\Delta r_{r c}\right)+\frac{1}{6}\left(2 \Delta r_{r a}-\Delta r_{r b}-\Delta r_{r c}\right) \cos \left(2 \theta_{r}\right) \\
& +\frac{\sqrt{3}}{6}\left(\Delta r_{r b}-\Delta r_{r c}\right) \sin \left(2 \theta_{r}\right) \\
r_{r 12}= & \left.-\frac{1}{6}\left(2 \Delta r_{r a}-\Delta r_{r b}-\Delta r_{r c}\right)\right) \sin \left(2 \theta_{r}\right)+\frac{\sqrt{3}}{6}\left(\Delta r_{r b}-\Delta r_{r c}\right) \cos \left(2 \theta_{r}\right) \\
r_{r 13}= & \left.\frac{1}{3}\left(2 \Delta r_{r a}-\Delta r_{r b}-\Delta r_{r c}\right)\right) \cos \left(\theta_{r}\right)-\frac{\sqrt{3}}{3}\left(\Delta r_{r b}-\Delta r_{r c}\right) \sin \left(2 \theta_{r}\right) \\
r_{r 22}= & \frac{1}{3}\left(\Delta r_{r a}+\Delta r_{r b}+\Delta r_{r c}\right)-\frac{1}{6}\left(2 \Delta r_{r a}-\Delta r_{r b}-\Delta r_{r c}\right) \cos \left(2 \theta_{r}\right) \\
& +\frac{\sqrt{3}}{6}\left(\Delta r_{r b}-\Delta r_{r c}\right) \sin \left(2 \theta_{r}\right) \\
r_{r 23}= & -\frac{1}{3}\left(\Delta r_{r a}+\Delta r_{r b}+\Delta r_{r c}\right) \sin \left(\theta_{r}\right)-\frac{\sqrt{3}}{3}\left(2 \Delta r_{r a}-\Delta r_{r c}\right) \cos \left(2 \theta_{r}\right) \\
r_{r 33}= & \frac{1}{3}\left(\Delta r_{r a}+\Delta r_{r b}+\Delta r_{r c}\right) \\
r_{r 21}= & r_{r 12} \\
r_{r 31}= & \frac{1}{2} r_{r 13} \\
r_{r 32}= & \frac{1}{2} r_{r 23}
\end{aligned}
$$

and the matrix $R_{d q}$ will be also affected, due to the broken bar fault as:

$\mathbf{R}_{\mathbf{b r}}{ }^{*}=\left[\begin{array}{cccc}r_{s} & 0 & 0 & 0 \\ 0 & r_{s} & 0 & 0 \\ 0 & -\omega_{r} / \omega_{s} L_{m} & r_{r q} & -\omega_{s} / \omega_{s} L_{r} \\ \omega_{r} / \omega_{s} L_{m} & 0 & \omega_{r} / \omega_{s} L_{r} & r_{r d}\end{array}\right]$

with:

$$
\begin{aligned}
& r_{r q}=r_{r}+r_{r 11} \\
& r_{r d}=r_{r}+r_{r 22}
\end{aligned}
$$

Therefore the matrices $\mathbf{A}^{*}, \mathbf{B}^{*}$ in the faulty case will become:

$$
\mathbf{A}^{*}=-\mathbf{R}_{\mathrm{br}}^{*} \mathbf{L}^{-1}
$$

with

$$
\mathbf{A}^{*}=\frac{1}{\delta}\left[\begin{array}{cccc}
-L_{r} r_{s} & 0 & L_{m} r_{s} & 0 \\
0 & -L_{r} r_{s} & 0 & L_{m} r_{s} \\
L_{m} r_{r q} & 0 & -L_{s} r_{r q} & w_{r} \delta \\
0 & L_{m} r_{r d} & -w_{r} \delta & -L_{s} r_{r d}
\end{array}\right]
$$

$$
\mathbf{B}^{*}=\mathbf{B}
$$

In the sequel the $q-d$ model of the induction motor is being transformed to a MIMO ARMA system that can be provided by:

$$
\left[\begin{array}{c}
i_{q s}^{\mathrm{aa}}(t) \\
i_{d s}(t) \\
\dot{i}_{q r}(t) \\
\dot{i}_{d r}(t)
\end{array}\right]=\left[\begin{array}{c}
\theta_{q s}(t) \\
\theta_{d s}(t) \\
\theta_{q r}(t) \\
\theta_{d r}(t)
\end{array}\right]^{T} \cdot\left[\begin{array}{c}
\Phi_{q s}(t) \\
\Phi_{d s}(t) \\
\Phi_{q r}(t) \\
\Phi_{d r}(t)
\end{array}\right]^{T}+\left[\begin{array}{c}
e_{q s}(t) \\
e_{d s}(t) \\
e_{q r}(t) \\
e_{d r}(t)
\end{array}\right]^{T}
$$

where $\theta_{j}(t)$ is the parameter vector sets and the subindex $j$ represents the current set that can be selected as one from: $[q s, d s, q r, d r]$. Moreover $\theta_{j}(t)$ contains the corresponding coefficients of the selected ARMA model and can be defined in the general case as:

$$
\theta_{j}^{T}(t)=\left[F_{j, 1}(t) \ldots F_{n, 1}(t), T_{j, 1}(t), \ldots, T_{m, 1}(t)\right]^{T}
$$

Where $n$ and $m$ are the orders of the numerator and denominator for each considered transfer function respectively. The regression vector $\Phi_{j}(t)$ is being formulated as:

$\Phi_{j}^{T}(t)=\left[-y_{j}(t-1), \ldots,-y_{j}(t-n), \ldots, u_{j}(t+m-n-1), \ldots, u_{j}(t-n)\right]$

In (30) corrupting noise effecting the measurements is also taken under consideration, while it is assumed that this noise sequence is bounded by $\gamma_{j} \in \mathfrak{R}^{+}$as:

$$
\gamma_{j}\left\|e_{j}(t)\right\|^{2} \leq 1, \forall t
$$

Finally the parameters in (31) have a direct relation with the motor's parameters in both the healthy and the faulty case. For example, for the case of $j=q s$ for the faulty case, these parameters can be defined as:

$$
\begin{aligned}
& T_{q s, 1}=\frac{L_{r}}{\delta}, \quad T_{q s, 2}=-b_{1}\left(a_{1}+a_{4}+a_{7}\right) \\
& T_{q s, 3}=b_{1}\left(a_{5}^{2}+a_{1} a_{4}+a_{1} a_{7}-a_{2} a_{6}+a_{4} a_{7}\right) \\
& T_{q s, 4}=b_{1}\left(a_{1} a_{5}^{2}+a_{1} a_{4} a_{7}-a_{2} a_{4} a_{6}\right. \\
& F_{q s, 1}=\left(2 a_{1}-a_{4}+a_{7}\right) \\
& F_{q s, 2}=a_{1}^{2}+a_{5}^{2}+a_{2} b_{2}+2 a_{1} a_{4}-a_{2} a_{3} \\
& +2 a_{1} a_{7}-a_{2} a_{6}+a_{4} a_{7} \\
& F_{q s, 3}=a_{1}^{2}\left(a_{7}-a_{4}\right)+2 a_{1} a_{5}^{2}-a_{1} a_{2}\left(a_{3}+a_{6}\right) \\
& +2 a_{1} a_{4} a_{7}-a_{2} a_{3} a_{7}-a_{2} a_{4} a_{6}+a_{2} b_{2}\left(a_{1}+a_{7}\right) \\
& F_{q s, 4}=a_{1}^{2} a_{5}^{2}+a_{2}^{2} a_{3} a_{6}-a_{1} a_{2}\left(a_{3} a_{7}+a_{4} a_{6}\right)+a_{2} b_{2}\left(a_{1} a_{7}\right) \\
& a_{1}=-\left(L_{r} r_{s}\right) / \delta \quad a_{4}=-\left(L_{s} r_{r q}\right) / \delta \quad b_{1}=L_{r} / \delta \\
& a_{2}=\left(L_{m} r_{s}\right) / \delta \quad a_{5}=\omega_{r} \quad b_{2}=-L_{m} / \delta \\
& a_{3}=\left(L_{m} r_{r q}\right) / \delta \quad a_{6}=\left(L_{m} r_{r d}\right) / \delta \quad a_{7}=-\left(L_{s} r_{r d}\right) / \delta
\end{aligned}
$$

\section{SET MEMBERSHIP FAULT IDENTIFICATION}

Set membership identification (SMI) refers to a class 
of techniques for estimating parameters of linear systems or signal models under a priori information that constrains the solutions to certain sets. The objective of the SMI technique is the determination of the feasible parameter set that contains the nominal parameter vector and is consistent with a linearly parameterizable model, the measurement data and the a priori known bounded noise-error. Due to the complexity in computing the feasible parameter set, the majority of the SMI methods aims at the determination of a more conveniently computable parametric set that outer bounds the feasible parameter set (Ljung, 1987; Deller, 1989).

The SMI technique is based on the Weighted Recursive Least Squares (WRLS) with a forgetting factor for identifying the $\hat{\theta}_{j}$ motor's parameters and can be formulated by the following double recursions (Guastafsson, 2001) in the sample instance $t$ and for the MIMO case $j$ as:

$$
\begin{aligned}
\hat{\theta_{j}}(t) & =\hat{\theta_{j}}(t-1)+K_{j}(t)\left(y_{j}(t)-\Phi_{j}^{T}(t) \theta_{j}(t-1)\right) \\
K_{j}(t) & =P_{j}(t-1) \Phi_{j}(t)\left(\lambda+\Phi_{j}^{T}(t) P_{j}(t-1) \phi_{j}(t)\right)^{-1} \\
P_{j}(t) & =\left(I-K_{j}(t) \Phi_{j}^{T}(t)\right) P_{j}(t-1) / \lambda \\
e_{j}(t) & =y_{j}(t)-\Phi_{j}^{T}(t) \theta_{j}(t-1) \\
G_{j}(t) & =\Phi_{j}^{T}(t) P_{j}(t-1) \Phi(t)
\end{aligned}
$$

In the SMI approach the initial bounds $\gamma$ for the corrupting noise $\varepsilon_{j}(t)$ are being re-calculated in every iteration. This optimization in the uncertainty description is evolving with the time, as the better the knowledge of the parameters is, the smaller these bounds are. To calculate the optimal value of $\lambda_{j}^{*}(t)$ for achieving convergence, the maximum positive root of the following equation should be extracted in each iteration:

$$
\begin{aligned}
F_{j}\left(\lambda_{j}\right) & =\alpha_{2, j} \lambda_{j}^{2}+\alpha_{1, j} \lambda_{j}+\alpha_{0, j} \\
\alpha_{2, j} & =(\ell+n-1) G_{j}^{2} \\
\alpha_{1, j} & \left.=\left(2 \ell+2 n-1+\gamma_{j} e_{j}^{2}\right)-\xi_{j} \gamma_{j} G_{j}\right) G_{j} \\
\alpha_{0, j} & =(\ell+n) *\left(1-\gamma_{j} e_{j}^{2}\right)-\xi_{j} G_{j} \gamma_{j} \\
\xi_{j}(t) & =\xi_{j}(t-1)+\frac{\lambda_{j}}{\gamma_{j}}-\frac{\lambda_{j} e_{j}}{1-\lambda_{j} G_{j}}
\end{aligned}
$$

with $\ell=m+1$. For founding the upper and lower boundary for the identified parameters, the uncertainty bound $\sigma_{j}(t)$, should be computed in every iteration. For delivering these bounds, the smallest orthotope that bounds the ellipsoidal uncertainty of the parameter and it is oriented parallel to the parameter coordinate axes and centered on the centroid of the ellipsoid is being calculated as:

$$
\sigma_{j}(t)=\sqrt{\operatorname{diag}\left(P_{j}(t)\right)}
$$

while the corresponding equation for the ellipsoids $\Omega_{j}^{e}(t)$ and the orthotopes $\Omega_{j}^{p}(t)$ can be calculated as (Deller, 1989):

$\left.\Omega_{j}^{e}(t)=\left\{\theta_{j}:\left(\theta_{j}(t)-\hat{\theta}_{j}(t)\right)^{T} \frac{C_{j}(t)}{\xi_{j}(t)}\right)\left(\theta_{j}(t)-\hat{\theta}_{j}(t)\right) \leq 1, j=1, \ldots, n+m\right\}$

$\Omega_{j}^{p}(t)=\left\{\theta_{j}:\left(\frac{1}{\left|\sigma_{j}(t)\right|}\right)\left(\theta_{j}(t)-\hat{\theta}_{j}(t)\right) \leq 1, j=1, \ldots, n+m\right\}$

with the orthotope's vertices $V_{j, o}^{n+m+1}(k), \quad o=$ $0, \ldots, 2^{n+m+1}-1$ are related to the $\sigma_{j}$ parameters as: $V_{j, o}^{p}(k)=\theta_{j}(t)+\left[\alpha_{j, n+m+1}^{*} \sigma_{j, n+m+1}(t), \ldots, \alpha_{j, 1} \sigma_{j, 1}(t)\right]^{T}$ where $\alpha_{j,(\cdot)}^{*}=\left\{\begin{array}{l}+ \text { when } a_{j,(\cdot)}=1 \\ - \text { when } a_{j,(\cdot)}=0\end{array}\right.$, and the covariance matrix is denoted as $C_{j}(t)=P_{j}(t)^{-1}$. The matrix $W(t)=\frac{C_{j}(t)}{\xi_{j}(t)}$ will represent how far the ellipsoid extends in each direction from the center of the ellipsoid $\hat{\theta}_{j}(t)$, while the volume ratio of the $j$ ellipsoid is being calculated by: $B_{j}(t)=\operatorname{det}^{-1}(W(t))$.

\section{FAULT DETECTION CONDITIONING}

In the case of a broken bar fault occurrence, the values of the identified parameters will be characterized by a jump and a constant drift from the converged nominal values of the healthy motor. This jump has a direct effect on the updated parameter's bounds, as after the event of the fault the following calculated uncertainty bounds will exceed the previous calculated bounds in the healthy case, while this situation is a direct indication of a fault occurrence. Based on the proposed SMI scheme, the following rules for the fault occurrence identification will be established as it is being depicted in Figure 1. In the presented approach it has been assumed that the SMI scheme is providing smooth value updates for the identified parameters.

If a $t_{1}$ time window is being defined, then after the convergence of the parameters, small changes in the identified values should be allowed, while a significant change might indicate the occurrence of a fault. For the ad-hoc defined bound $B_{1}$ the first fault detection condition is being formulated as:

$[\underline{\text { Condition 1 }}]\left|\theta_{j}^{o}(k)-\overline{\hat{\theta}_{j}\left(k-t_{1}: k\right)}\right| \geq B_{1}$

where $\theta_{j}^{o}(k)$ denotes the converged identified parameter and the notation - represents a moving average time window of length $t_{1}$. Two additional rules can be defined, that are related with the volumes of the bounding ellipsoids $\Omega_{j}^{e}$ or orthotopes $\Omega_{j}^{p}$. The aim is to track the corresponding volumes of the bounding ellipsoid/orthotope and allow only small 


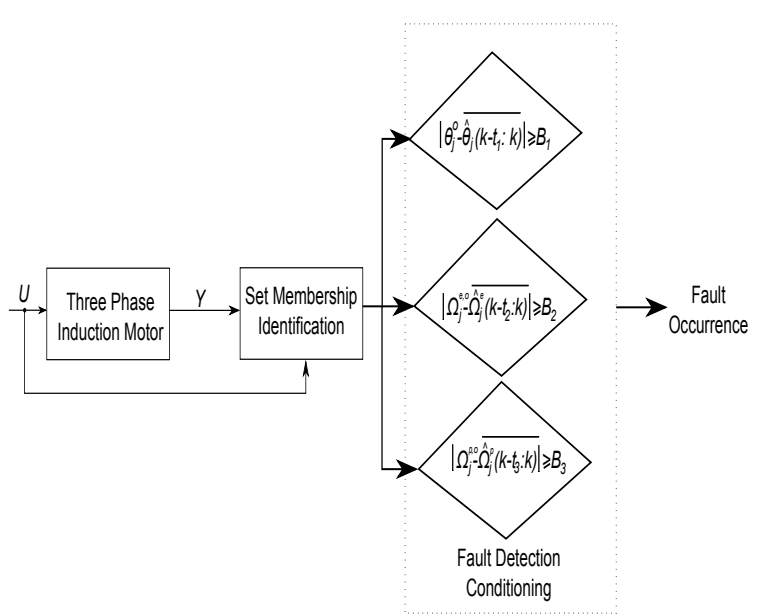

Figure 1: Faults detection scheme.

changes, as in the opposite case, this should generate a fault. By defining two ad-hoc boundaries as $B_{2}$ and $B_{3}$ and two additional time windows $t_{2}, t_{3}$, the following additional fault detection conditions can be formulated:

\section{[Condition 2] $\left|\Omega_{j}^{e, o}(k)-\overline{\Omega_{j}^{e}\left(k-t_{2}\right): k}\right| \geq B_{2}$ \\ [Condition 3] $\left|\Omega_{j}^{p, o}(k)-\overline{\Omega_{j}^{p}\left(k-t_{3}: k\right)}\right| \geq B_{3}$}

Where $\Omega^{e, o}, \Omega^{p, o}$ are the converged values and conditions (2) and (3) are complimentary and depending on the processing capabilities and the accuracy that we would like to achieve. In general, condition (2) is more accurate that condition (3), as the orthotope is bounding the uncertainty described by the ellipsoid, but this approach has the drawback that it is more computational demanding.

\section{SIMULATION RESULTS}

The suggested scheme for fault detection is being evaluated on a model of three phase induction motor having the parameters depicted in Table 1.

Table 1: Induction Motor Parameters.

\begin{tabular}{|l|c||c|c|}
\hline Pole Numbers & 4 & $r_{s}$ & 0.0616 per unit \\
\hline Input Voltage & $240 \mathrm{~V}$ & $r_{r}$ & 0.0753 per unit \\
\hline Frequency & $50 \mathrm{~Hz}$ & $\mathrm{~J}$ & $0.00155 \mathrm{Kg} . \mathrm{m}$ \\
\hline$L_{r}$ & 0.019 per unit & $L_{s}$ & 0.019 per unit \\
\hline$L_{m}$ & 0.01833 per unit & $N_{b}$ & 28 \\
\hline
\end{tabular}

The first simulation results will focus in presenting the effect of one broken bar fault occurrence on the rotor's current, torque and angular speed, as it is

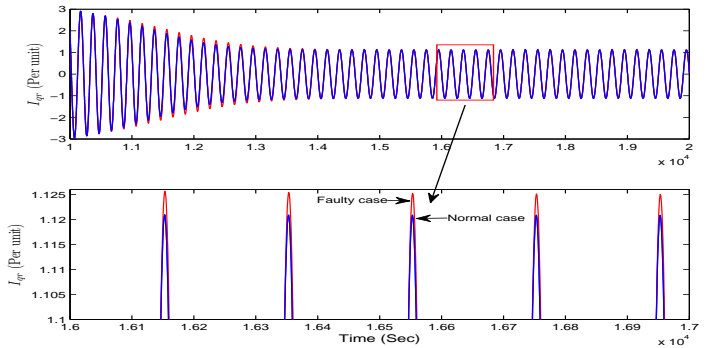

Figure 2: The rotor's currents in the normal and faulty case.

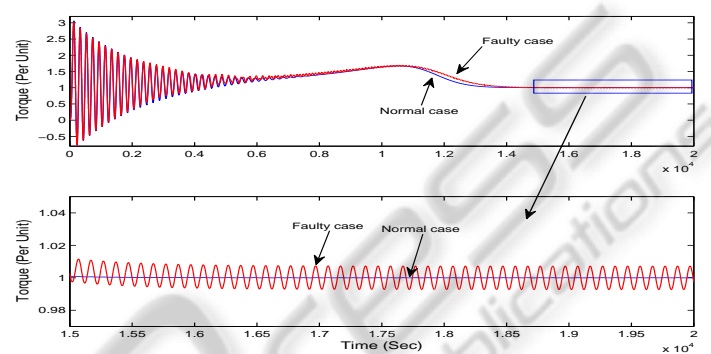

Figure 3: The torque of the motor in the normal and faulty case.

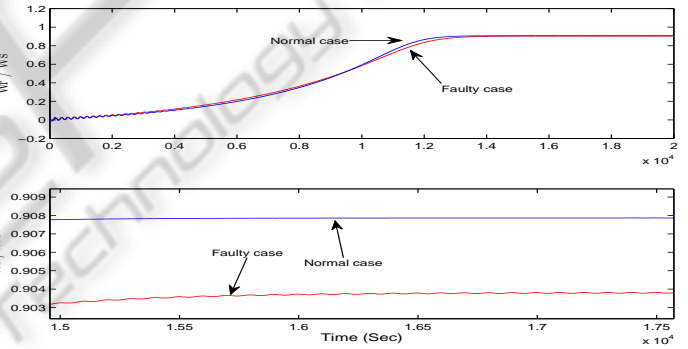

Figure 4: The rotor angular speed of the motor in the normal and faulty case.

being presented in Figures (2-4) respectively. For the rotor's current, same results have been also obtained as in Figure (2).

From the obtained results, it can be observed that the small change in the values of the currents can be distinguished, where the rotor's current $I_{q r}$ in the faulty case will be increased from the corresponding value in the healthy case. In Figures (3 and 4), it is also depicted that the fault is affecting the steady state values of the rotor's torque and speed respectively. In the case of the motor's torque oscillations are taking place, while the amplitude of these oscillations is being increased with respect to the number of the faulty broken bars. In the second case, the speed is also been decreased, while oscillations are also evident.

In the sequel, results from applying the proposed SMI scheme for broken bar fault detection are going to be presented. Figures (5), and (6) present the convergence of the SMI identification (with the uncertainty bounds) of the motor's parameters in the 

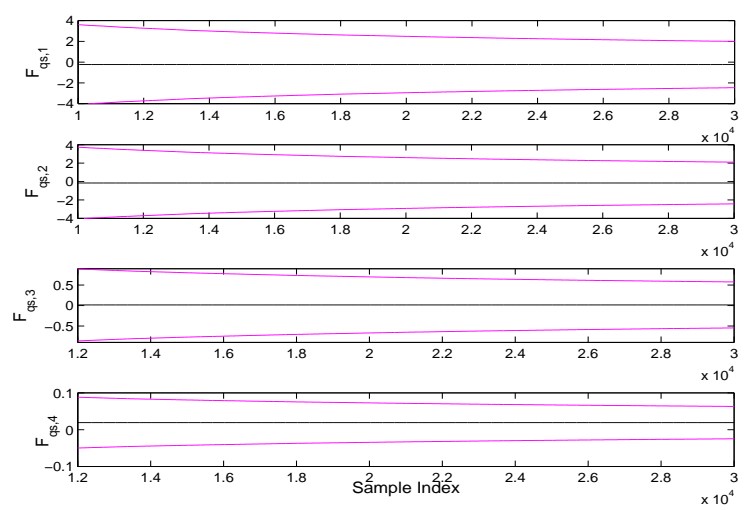

Figure 5: SMI based identified parameters for $F_{i_{q s}}$ and corresponding uncertainty bounds.
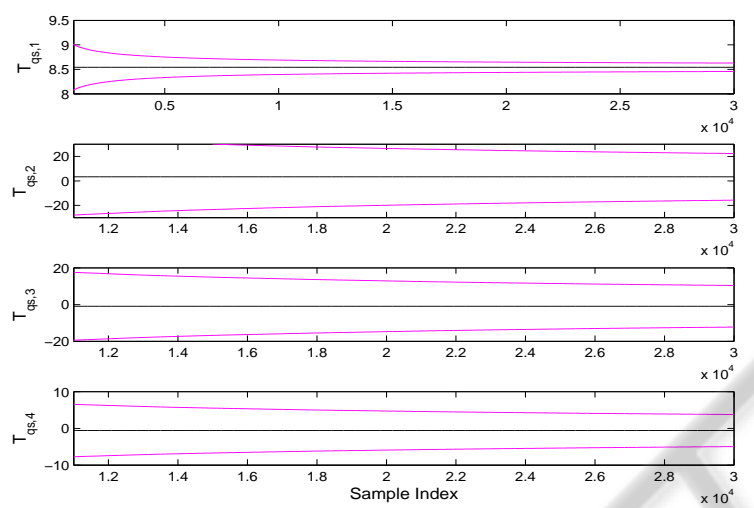

Figure 6: SMI based identified parameters for $T_{i_{q s}}$ and corresponding uncertainty bounds.

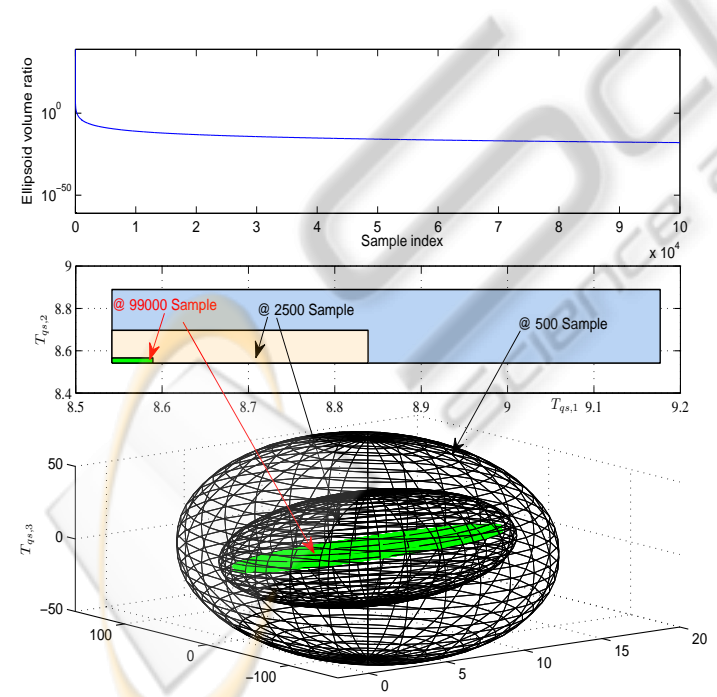

Figure 7: Orthotope and ellipsoid change with sample index.

healthy case, only for the case of having as input $V_{q s}$ and output $i_{q s}$, while similar results have been obtained for the rest of the motor's parameters, but due to space limitations, those graphs have been omitted.

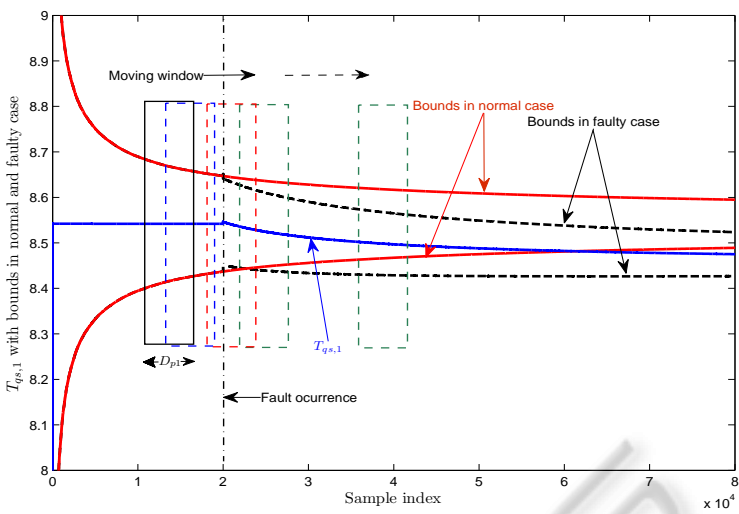

Figure 8: Convergence of the $T_{q s, 1}$ parameter and corresponding bounds before and after the occurrence of the fault.

As it can be observed from these figures the uncertainty bounds are starting from a large value and in the sequence, as the identification procedure is evolving and the identified parameters are close to the nominal values, those bounds are being decreased, until it reach their steady state value. Finally, in Figure (7) the convergence of the ellipsoid volume and the convergence of the orthotope's volume is being presented and the corresponding bound sets of uncertainty are being presented at different time indexes, where the evolution of the volume has been highlighted with respect to the sample index.

In Figure 8, the case of a fault occurred at 20000 samples (Sampling time $T_{s}=0.01$ ) is being presented. Due to this fault a jump in the identified parameters is taking place that affects the presented conditions for fault detection. More specifically, the uncertainty intervals are changing (due to the drift in the identified model) and this leads in a bounds violation event, which indicates the existence of the broken rotor fault. Similar graphs can also be extracted for all the identified parameters of the motor and without loosing generality, in Figure 8, only the results for $T_{q s, 1}$ are being presented.

The effectiveness of the identification scheme can also be examined by inspecting the volume of the bounding ellipsoid. As it is being displayed in Figure (7) the ellipsoid volume will be minimized as the identified model matches the real one. In the even of a fault, the identified values are being drifted to small values (resulting from the faulty model representation) and thus is why the bounds are being kept on monotonically being decreased. In the case of a rotor's broken bar, the operation of the motor is still unaffected, and the result due to the fault is a small parametric drift and this is why a the simple inspection of the identified parameters in not the only factor that should be monitored for fault identification, but 

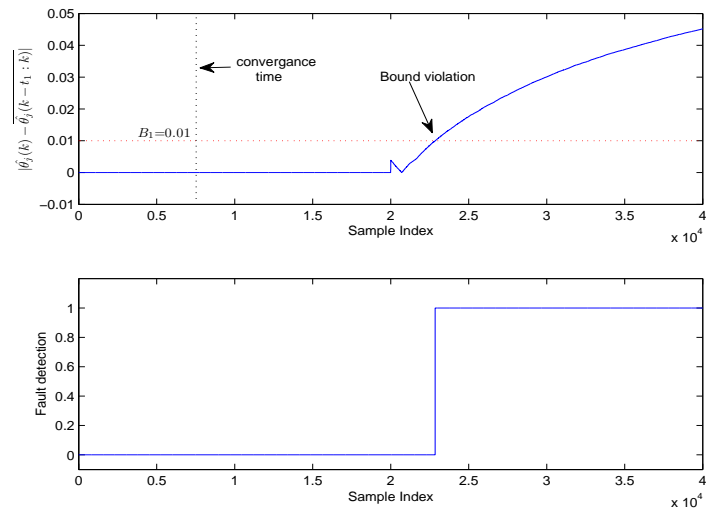

Figure 9: Fault detection after $1^{s t}$ condition monitoring.

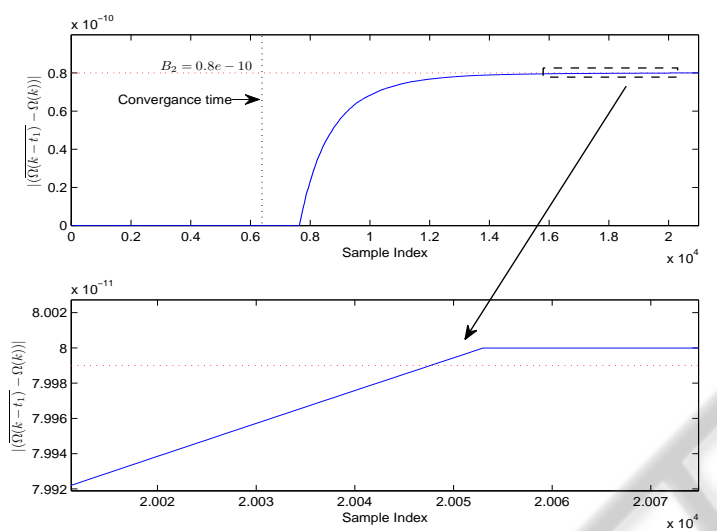

Figure 10: Fault detection after $2^{\text {nd }}$ condition monitoring/condition2.

is should be combined with the second or third condition for the ellipsoid or the orthotope's volume. In the examined case, the occurrence of the fault has caused a jump on the ellipsoid's volume convergence, which was bigger than the a priori defined bound and thus the algorithm is able to trigger the even of the fault.

The time evolutions of the fault detection conditions are being presented in Figures (9) and (10) where the convergence time for the identified parameters is also being displayed, along with the bound violation for the conditions (1) and (2).

\section{CONCLUSIONS}

In this article a fault detection scheme for broken rotor bar fault detection has been presented in the case of a three phase induction motor. The three phase induction motor has been modeled in the equivalent two phase motor $(q-d)$ space, while the modeling of the faulty case has been also formulated. The motor has been identified by the utilization of the SMI algorithm that has the merit of identifying both the parameters of the motor as also providing uncertainty safety bounds by calculating ellipsoids/orthotopes, which bounds the systems parameter vector. The obtained results present the efficiency of the proposed conditions to capture the event of the fault.

\section{REFERENCES}

Bachi, S., Tnani, S., Trigeassou, J., and Champenois, G. (2006). Diagnosis by parameter estimation of stator and rotor faults occurring in induction machines. IEEE Transactions on Industrial Electronics, 53(3):963-973.

Bellini, A., Filippetti, F., Franceschini, G., Tassoni, C., and Kliman, G. (2001). Quantitative evaluation of induction motor broken bars by means of electrical signature analysis. IEEE Transactions on Industry Application, 37(5):1248-1255.

Chen, S. and Zivanovic, R. (2009). Modelling and simulation of stator and rotor fault conditions in induction machines for testing fault diagnostic techniques. European Transactions On Electrical Power, 20:611 -629 .

Deller, J., Nayeri, M., and Odeh, S. (1993). Least-Square Identification with Error Bounds for Real-Time Signal Processing and Control. Proceedings of the IEEE, 81(6):815-849.

Deller, J. R. (1989). Set membership identification in digital signal processing. IEEE ASSP Magazine, 6(4):4-20.

Guastafsson, F. (Sep.2001). Adaptive Filtering and Change Detection.

Kumar, S., Prakash, J., and Kumar, S. S. (2011). Detection of broken rotor bars in induction motor using derivative free kalman filters. International Conference on Process Automation, Control and Computing (PACC), 2011, pages $1-7$.

Lee, R., Pillay, P., and Harley, R. (1985). Dq reference frames for the simulation of induction motors. Electric Power Systems Research, 8:15 -26.

Ljung, L. (1987). System Identification Theory For The User. Prentice-Hall, Englewood Cliffs, NJ.

Nandi, S. and Toliyat, H. (2005). Condition monitoring and fault diagnosis of electrical machines-a review. IEEE Transactions on Energy Conversion, 20(4):719-729.

Sandhu, K. and Pahwa, V. (2009). Sumilation study of three phase induction motor with variation in moment of inertia. ARPN Journal of Enginering and Apllied Sciences, 4(5):72-77.

Santos, P. and Lubiny, T. (2010). A simplified induction machine model to study rotor broken bar effects and for detection. European Transactions On Electrical Power, 20:611.

Vas, P. (1992). Electrical Machines and Drives. 